\title{
Narcissism between facets and domains. The relationships between two types of narcissism and aspects of the Big Five
}

\author{
Marcin Zajenkowski $^{1}$ (D) $\cdot$ Kinga Szymaniak $^{1}$
}

Published online: 23 January 2019

(C) The Author(s) 2019

\begin{abstract}
Grandiose and vulnerable narcissism have distinct personality profiles. Specifically, grandiose narcissism correlates positively with Extraversion and negatively with Neuroticism, whereas vulnerable narcissism showed a reverse associations with these traits. Additionally, both types of narcissism have common antagonistic core as they are negatively related to Agreeableness. Although correlations between narcissism and basic personality traits are well-documented, researchers focused mainly on broad, higher order factors which reflect a very wide spectrum of behavior. To better understand the nature of narcissism and its personality correlates, we examined the association of narcissisms with 10 aspects of the Big Five. Aspects are located between domains and facets in the personality hierarchy. The results of the current study $(n=437)$ indicated that grandiose narcissism was more differentiated within all of the Big Five traits. Specifically, grandiose narcissism correlated mainly positively with Assertiveness (from Extraversion), and Intellect (Openness/Intellect), and negatively with Politeness (Agreeableness), Industriousness (Conscientiousness) and Withdrawal (Neuroticism). Vulnerable narcissism showed less differentiated correlations within broad domains, except for Openness/Intellect. Surprisingly, individuals with high vulnerable narcissism exhibited a high level of Openness. The results of the current study contribute to our understanding of the two types of narcissism and their personality correlates.
\end{abstract}

Keywords Big Five $\cdot$ Grandiose narcissism $\cdot$ Narcissism $\cdot$ Personality $\cdot$ Vulnerable narcissism

\section{Introduction}

Recent studies suggest the existence of two forms of narcissism: grandiose and vulnerable (Miller et al. 2011; Pincus et al. 2009). Both types of narcissism share common features, such as self-centeredness, sense of entitlement, and disregard of others (e.g., Miller et al. 2011); they differ substantially in many other respects. Specifically, grandiose narcissism is characterized by an inflated positive self-image, high self esteem, exhibitionism, attitudes of entitlement, a tendency toward exploitativeness, and the need to be admired by others (Miller et al. 2011; Pincus et al. 2009). Vulnerable narcissism, on the other hand, is characterized by defensiveness, avoidance, insecurity, hypersensitivity, vulnerability, low self-esteem, high anger and hostility (Miller et al. 2011; Pincus

Marcin Zajenkowski

Zajenkowski@psych.uw.edu.pl

Faculty of Psychology, University of Warsaw, Warsaw, Poland et al. 2009). The extant studies revealed that both types of narcissism correlate differentially with a number of psychological outcomes including affective functioning, interpersonal behavior, and psychological adjustment. Grandiose narcissism is related to positive psychological profiles like positive affect, a high level of well-being, and low levels of loneliness, sadness, depressive, and anxious feelings (e.g., Sedikides et al. 2004), whereas vulnerable narcissism is associated with higher levels negative emotionality, including depression, anxiety, anger, shame, or envy (see Czarna et al. 2018). Vulnerable narcissists, in comparison to grandiose ones, manifest more difficulties in emotion regulation and sympthoms of borderline personality disorders (Miller et al. 2011).

The two types of narcissism were found to be related to basic personality traits, such as the Big Five (e.g. Miller et al. 2011). Individuals with high grandiose narcissism are more extraverted and stable (low neurotic; Jauk et al. 2017; Miller et al. 2011) and score higher on Openness/Intellect (Zajenkowski et al. 2016). In contrast, vulnerable narcissism is associated with high negative emotionality, including high levels of anxiety, anger, or depression (Miller et al. 2011), and Miller et al. (2018b) showed that the lion's share of the 
vulnerable narcissism variance might be explained by Neuroticism. Additionally, vulnerable narcissism is linked with introversion (Miller et al. 2011). Finally, Agreeableness is negatively correlated with both types of narcissism (e.g. Miller et al. 2011). So far researchers have focused mainly on broad, higher order factors which reflect a very wide spectrum of behavior. To better understand the nature of narcissism it would be valuable to examine more specific aspects of broad personality domains. For instance, research by Miller et al. (2011) found that there were differences between grandiose and vulnerable narcissism in their correlations with the Big Five facets. However, most widely known facets of the Revised NEO Personality Inventory (NEO-PI-R; Costa and McCrae 1992b) have been criticized by some researchers, who proposed instead more empirically rooted aspects of the Big Five (DeYoung et al. 2007). In the present study, we focus on the relationship between grandiose and vulnerable narcissism and 10 aspects of the Big Five described by DeYoung et al. (2007). We provide arguments that in some cases, each type of narcissism might be similarly associated with two aspects within one domain, while in other cases they may show a stronger correlation with one aspect of a broader trait.

\section{Two Types of Narcissism and Aspects of the Big Five}

The most popular classification system for personality traits — the Big Five personality model - delineates five broad personality domains: Neuroticism, Extraversion, Agreeableness, Conscientiousness and Openness (e.g., Costa and McCrae 1992b; Goldberg 1992). Initially, these traits were perceived as orthogonal factors and unitary constructs (Costa and McCrae 1992a). Then, Costa and McCrae (1992b) proposed a two-level structure of personality traits, where each domain was divided into six facets. However, some of the research calls into question this solution, showing that there might be more levels of personality hierarchy (Digman 1997). For instance, higher order factors of the Big Five (meta-traits) have been identified and described by Digman (1997) as stability (low Neuroticism and high Agreeableness and Conscientiousness) and plasticity (high Openness and Extraversion). More recently, DeYoung et al. (2007) suggested the existence of an intermediate level of trait organization located between facets and domains. They provided a detailed characterization of the 10 aspects of the Big Five by analyzing a broad range of personality facets from the two popular Big Five inventories: the NEO-PI-R and the Abridged Big Five-Dimensional Circumplex from the International Personality Item Pool (AB5C-IPIP). In both instruments the facets were distinguished in different ways. In the NEO-PI-R the facets were derived theoretically, based on a review of the literature (Costa and McCrae 1992b), whereas the facet structure of the AB5C-IPIP was based on a circumplex model of personality traits (Goldberg 1992). DeYoung et al. (2007) suggested that there are two distinct aspects located between each broad domain and its narrow facets. They state that aspects are related but separable trait dimensions, more parsimonious and less arbitrary than facets. However, it needs to be acknowledged that the authors themselves made several arbitrary decisions (e.g. using particular personality instruments, factor-analysis method etc.) in order to obtain exactly two aspects within each domain. Nevertheless, they also indicated some evidence that the aspects within each of the Big Five are likely to have partially distinct biological substrates, environmental influences, and effects (DeYoung 2015). For instance, it has been found that two genetic factors are responsible for the shared variance of the facet scales within each of the Big Five trait in the NEOPI-R (Jang et al. 2002). Additionally, in case of some domains, e.g. Openness, it has been shown that the aspects manifest different neurological (DeYoung et al. 2009), cognitive (DeYoung et al. 2012) and affective (Zajenkowski and Matthews 2019) correlates. Thus, 10 aspects of the Big Five seem to be useful to better understand the nature of each type of narcissism. Below, we describe aspects of the Big Five and their potential connection to narcissism.

\section{Neuroticism}

Within Neuroticism, DeYoung et al. (2007) distinguished two factors labeled Volatility and Withdrawal. Volatility encompasses emotional lability, irritability, anger, and difficulty controlling emotional impulses. It is related to an active response to threats, leading to the outward expression of negative affect (DeYoung 2015). Withdrawal reflects passive avoidance, increased depression, vulnerability, anxiety and negative affect directed inward (DeYoung 2015). Existing literature shows that vulnerable narcissism correlates positively with a wide range of phenomena related to negative emotionality including anxiety and depression (Miller et al. 2011), but also anger and aggressiveness (Krizan and Johar 2015; Czarna et al. 2018). Thus, one can expect that vulnerable narcissism would be positively correlated with both Volatility and Withdrawal (H1). In the case of grandiose narcissism we expect a negative relation with Withdrawal, relatively stronger than with Volatility (H2). Those high on grandiose narcissism exhibit a low level of anxiety and depression (Sedikides et al. 2004), but, on the other hand, tend to show a moderate level of anger (Krizan and Johar 2015).

\section{Extraversion}

In the case of Extraversion, DeYoung et al. (2007) identified two factors labeled Assertiveness (agency, social dominance, the tendency toward drive, social status, leadership, and high 
motivation to attain desired goals) and Enthusiasm (sociability and the tendency to experience positive emotions associated with anticipation of reward). We hypothesize that grandiose narcissism will be positively associated with both aspects of Extraversion, but we expect stronger correlation with Assertiveness (H3). The main characteristic of grandiose narcissism is agency and social dominance (Campbell and Foster 2007), the core features of Assertiveness. It was found that individuals high on grandiose narcissism tend to be more assertive and dominant in interpersonal relations (Pincus et al. 2009). They use assertive self-presentation tactics (Hart et al. 2017) and aggression in order to resolve problems (Miller et al. 2011). Moreover, the Assertiveness subscale from the NEO-PI-R was the strongest correlate $(r=$ 0.63 ) of grandiose narcissism among the Extraversion facets (Miller et al. 2011). Additionally, grandiose narcissism is associated with high levels of well-being and positive emotionality (Sedikides et al. 2004), which would suggest a positive relation with the Enthusiasm aspect. However, the latter also includes sociability, understood as warm and friendly interactions, which is not characteristic for narcissists who show rather increased exploitativeness in interpersonal functioning (Czarna et al. 2016). Thus, we expect a positive correlation between grandiose narcissism and Enthusiasm, but weaker than with Assertiveness.

We predict that vulnerable narcissism will be negatively correlated with both Assertiveness and Enthusiasm (H4). Individuals scoring high on vulnerable narcissism show low positive affect, avoidance of close relationships and, as a result, limited social networks (Miller et al. 2011). These findings suggest rather low Enthusiasm among vulnerable narcissists. There is also some evidence suggesting that vulnerable narcissists would score low on Assertiveness. Lannin et al. (2014) found that vulnerable narcissists are more likely to express their attitude when there is low accountability for their actions, such as when one can remain relatively anonymous. Moreover, vulnerable narcissists seem to hold a fatalistic view, that is an orientation of helplessness, and the feeling of little influence on their life (Zajenkowski et al. 2016).

\section{Agreeableness}

In the Agreeableness domain, two aspects were described by DeYoung et al. (2007): Compassion (compassionate emotional affiliation with others, and relatively automatic emotional processes, including empathy, caring, and concern for others) and Politeness (consideration of and respect for others' needs and desires, and low tendency toward competitive attitude, lack of cooperation, and being rude and aggressive). We predict that grandiose narcissism will be negatively associated with both aspects of Agreeableness; however, the correlation with Politeness will be higher than with Compassion (H5). Grandiose narcissists hold inflated self-views, are arrogant and boastful, and have little regard for others (Campbell and Foster 2007). All these characteristic would suggest a strong negative correlation with Politeness. Although lack of empathy and compassion for others is thought to be a key characteristics of grandiose narcissism, empirical data provide rather mixed results in this area (see Czarna et al. 2018). Therefore, we expect that the association between grandiose narcissism and Compassion will be negative but less pronounced than with Politeness.

One may expect that vulnerable narcissism will be associated negatively with both aspects of Agreeableness, but, in contrast to grandiose narcissism, the relation will be relatively stronger with Compassion than Politeness (H6). Vulnerable narcissism has been found to correlate with a low level of empathy (e.g. Czarna et al. 2018). Moreover, it has been suggested that individuals who are high on grandiose narcissism may be antagonistic for instrumental reasons related to status and dominance, whereas individuals high on vulnerable narcissism may behave in hostile and distrustful ways due to heightened affective dysregulation. In fact, it is possible that vulnerable narcissists are more likely to conceal negative emotions and be kind towards others in order to not be criticized (Lannin et al. 2014).

\section{Openness/Intellect}

The compound label "Openness/Intellect" reflects the longrunning debate about whether to label this trait "Openness to experience" or "Intellect" (DeYoung et al. 2007). Intellect reflects intellectual engagement with abstract and semantic information; it correlates with IQ, and working memory, whereas Openness reflects engagement with sensory and perceptual information and thus involves aesthetic interests and fantasy proneness (DeYoung et al. 2012). Openness has been linked to creativity, implicit learning and automatic detection of patterns in sensory experience (DeYoung et al. 2012).

We expect that grandiose narcissism will be positively linked to Intellect and that this association will be relatively stronger than with Openness (H7). Intellect reflects the tendency to engage easily with abstract information; however, many of the items within this scale describe self-reported abilities, e.g., "Am quick to understand things" etc. (DeYoung et al. 2007). Numerous investigations have shown that grandiose narcissists tend to overestimate their positive qualities, especially in the agentic domain, such as cognitive abilities (Dufner et al. 2012; Gabriel et al. 1994; Zajenkowski and Czarna 2015). Thus, one may suspect that grandiose narcissists will score higher on Intellect due to their inflated narcissistic self-image. Indeed, existing data show a positive association between grandiose narcissism and Intellect (Zajenkowski et al. 2016) and Ideas from the NEO-PI-R (Miller et al. 2011). When it comes to Openness, there are 
no theoretical or empirical reasons to link it with grandiose narcissism.

Vulnerable narcissism is associated with variables, such as Neuroticism (Miller et al. 2018b), that predict underestimation of one's abilities (Chamorro-Premuzic and Furnham 2004), which would suggest its negative association with Intellect. However, the results of prior investigations show no relations between vulnerable narcissism and Intellect (Zajenkowski et al. 2016) nor with the NEO-PI-R Openness facets. Thus, we did not formulate any directional hypothesis with respect to Openness/Intellect and vulnerable narcissism.

\section{Conscientiousness}

The two factors within Conscientiousness were identified as Industriousness (need for achievements, self-discipline and the tendency to work effectively without being distracted) and Orderliness (neatness, perfectionism, and attention to rules; DeYoung et al. 2007). The existing data show no significant relationship between Conscientiousness and narcissism (Miller et al. 2011; Zajenkowski et al. 2016). Thus, we did not formulate hypotheses regarding the relation between the Conscientiousness aspects and narcissism.

\section{Method}

\section{Participants}

There were 437 (270 female) participants, who were recruited via publicly accessible social networking websites. Their mean age was 23 years $(S D=5.96)$. Participants were asked to complete a set of questionnaires some of which are not relevant for the current study. All subjects gave their informed consent for the release of their test scores for research purposes, and all were offered feedback on general results of the study. The dataset analysed during the current study is available at osf.io/xchju.

\section{Measures}

Vulnerable narcissism was measured with the Hypersensitive Narcissism Scale by Hendin and Cheek (1997) in the Polish translation (see Czarna et al. 2014). It consists of 10 items with a Likert-like response scale from 1 (strongly disagree) to 5 (strongly agree).

Grandiose narcissism was assessed with the validated Polish adaptation (Bazińska and Drat-Ruszczak 2000) of the Narcissistic Personality Inventory (Raskin and Hall 1979). The items were selected basing on the factor analysis (loadings exceeding .40) and represented the following subscales of the NPI: Authority (11 items), Self-Sufficiency (7), Vanity (5), and Exhibitionism (11). Respondents rated their degree of endorsement of each statement using a Likert-type response format, from 1 (does not apply to me) to 5 (applies to me). The sum of items creates an index of grandiose narcissism. It has been shown recently that the Likert format NPI manifests similar construct validity and slightly increased reliability to the original forced-choice format (Miller et al. 2018a).

The Big Five was assessed with Polish adaptation (Strus et al. 2014) of the International Personality Item Pool - Big Five Aspect Scale (BFAS; DeYoung et al. 2007). This is a 100-item measure of trait domains (Agreeableness, Conscientiousness, Extraversion, Neuroticism, and Openness/Intellect) and the two lower-order aspects of each domain (Volatility, Withdrawal, Compassion, Politeness, Industriousness, Orderliness, Enthusiasm, Assertiveness, Intellect, and Openness). Participants are asked to rate how much the statements are related to them on a Likert-type scale, from 1 (strongly disagree) to 5 (strongly agree).

\section{Results}

First, we tested the correlations between two variants of narcissism and personality traits including domains and aspects (Table 1). Both types of narcissism correlated with personality traits in a comparable way to previous research (e.g. Miller et al. 2011). Specifically, vulnerable narcissism was positively correlated with Neuroticism, and negatively associated with Agreeableness and Extraversion. Grandiose narcissism correlated positively with Extraversion, Openness/Intellect and Conscientiousness, and negatively with Neuroticism and Agreeableness.

Subsequently, we compared the associations between each type of narcissism and aspects of personality within each domain. Therefore, we tested for the difference between two dependent correlations (Steiger 1980). Figure 1 illustrates graphically the patterns of correlations. We found that vulnerable narcissism was significantly and positively correlated with both aspects of Neuroticism and that correlations did not differ from one another $(z=, 215, p=0.820)$, which supported H1. In addition, consistent with $\mathbf{H 2}$, grandiose narcissism was significantly and negatively correlated with Withdrawal, and this correlation was stronger than with Volatility $(z=5.85, p<0.001)$. Grandiose narcissism correlated positively with both aspects of Extraversion; however, the association was stronger for Assertiveness $(z=10.46, p<0.001)$, which supported H3. Vulnerable narcissism was equally negatively related to both Assertiveness and Enthusiasm $(z=-1.90 ; p=0.060 ;$ H4). The results also supported H5; grandiose narcissism was negatively correlated with both aspects of Agreeableness. However, the correlation with Politeness was significantly stronger $(z=6.26$; $p<0.001)$. The correlation of vulnerable narcissism with both aspects of Agreeableness was negative and the magnitudes of these correlations were similar $(z=.261 ; p=0.79)$. Thus, H6 


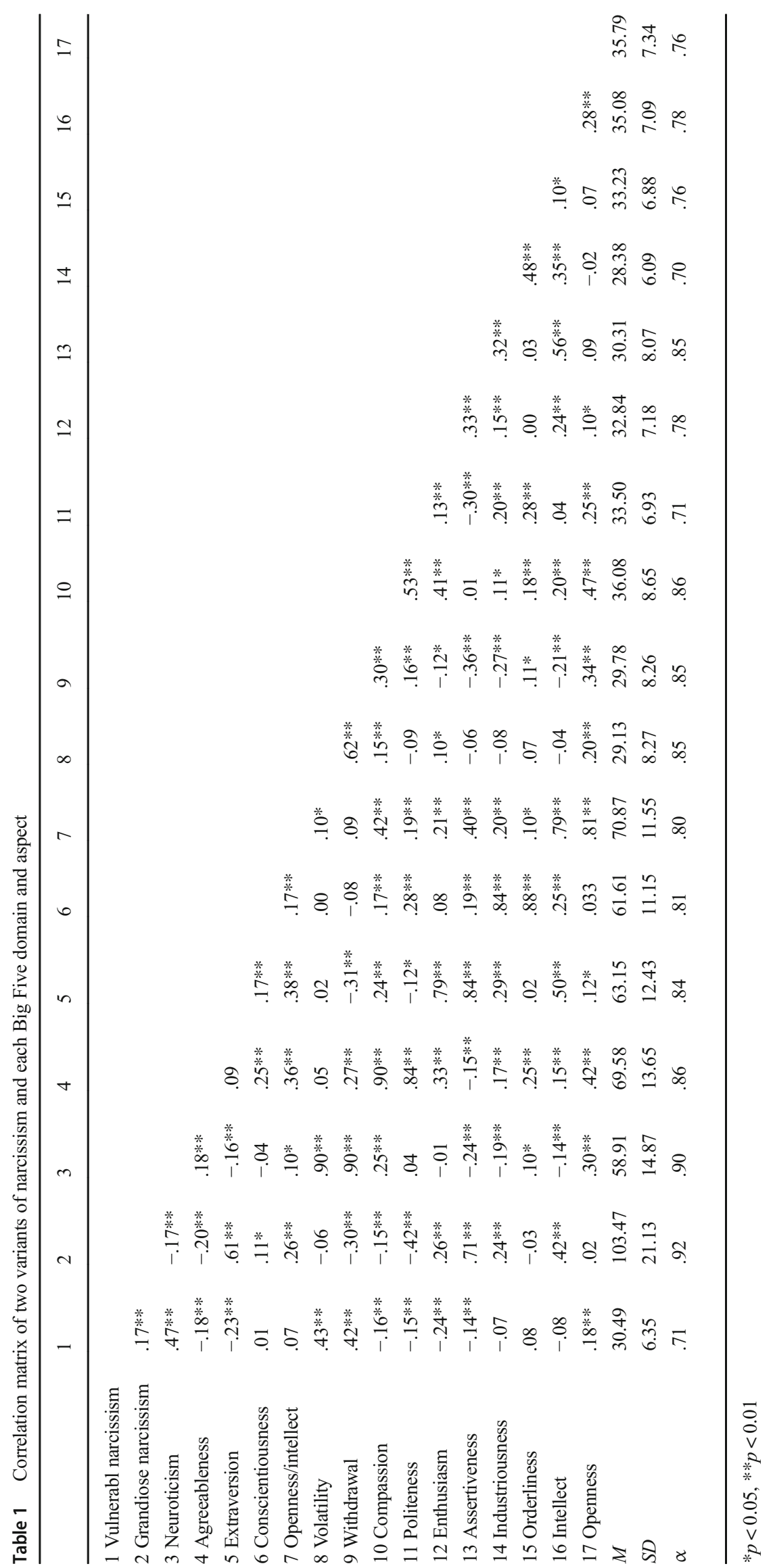




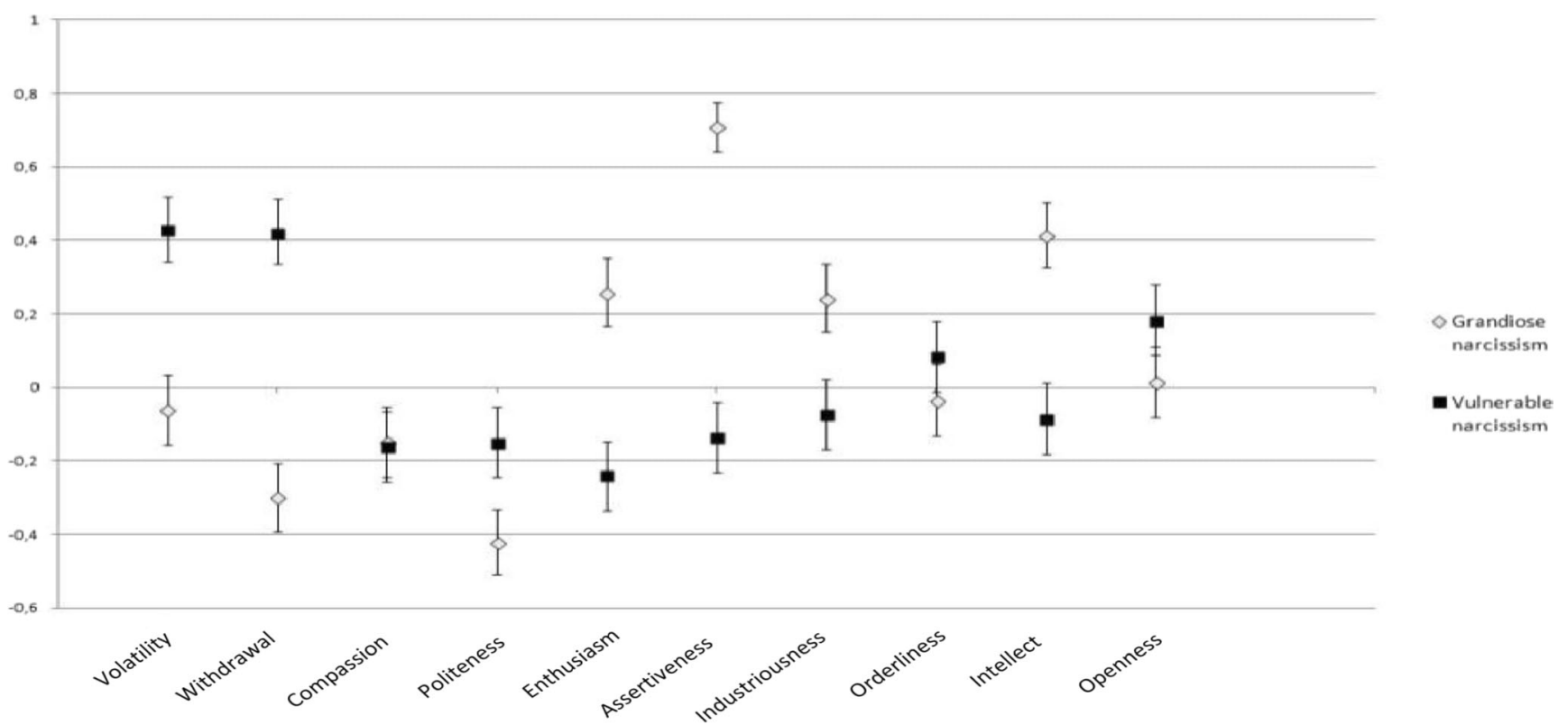

Fig. 1 Illustration of the correlation coefficients for the relationship between two variants of narcissism and 10 aspects of the Big Five

was only partially supported, because we expected a higher association with Compassion. Finally, $\mathbf{H} 7$ was also supported: grandiose narcissism correlated positively with Intellect and this association was stronger than with Openness $(z=7.40$; $p<0.001)$.

There were also some interesting results that we did not expect. First, Openness correlated positively with vulnerable narcissism, and the magnitude of this correlation differed from the association with Intellect $(z=-4.69 ; p<0.001)$. Second, Industriousness correlated positively with grandiose narcissism, and this association was stronger than with other aspects of Conscientiousness, i.e., Orderliness $(z=5.75$; $p<0.001)$.

Additionally, we examined partial correlations between each type of narcissism (controlling for the other narcissism) and Big Five traits and aspects (Table 2). The results revealed similar associations between narcissisms and personality as zero-order correlations (Table 1).

Finally, we compared the personality profiles between two types of narcissism. First, we calculated a double-entry intraclass correlation recommended by McCrae (2008) that allows the examination of the degree to which the grandiose and vulnerable narcissisms' personality profiles share absolute similarity. In this method, each element (here correlations with personality aspects) in the paired profile is entered twice but in reversed order across rows. In the current study, we obtained a negative correlation between profiles $(r=-.38)$ which suggests a high mismatch between narcissisms. In the last step we tested correlation differences between narcissisms' associations with Big Five aspects. The analysis (Table 3) indicated that grandiose and vulnerable narcissisms differed with respect to eight aspects with Withdrawal, Assertiveness and Intellect showing the biggest differences. We did not observe differences only in the case of Compassion and Orderliness.

\section{Discussion}

We found that grandiose and vulnerable narcissism were associated with personality aspects, i.e. lower-order factors of

Table 2 Partial correlations between each type of narcissism and personality domains and aspects with exclusion of the influence of the second narcissism

\begin{tabular}{lllllllllllllllllllll}
\hline & NEU & AGR & EXT & CON & OP/ & VOL & WITH & COM & POL & ENT & ASR & IND & ORD INT & OPE \\
\hline Vulnerable narcissism & $.52 * *$ & $-.14 * *$ & $-.42^{* *}$ & -.01 & .02 & $.45^{* *}$ & $.50^{* *}$ & $-.14^{* *}$ & -.09 & $-.30^{* * *}$ & $-.36^{* *}$ & $-.12^{*}$ & .09 & $-.17 * *$ & $.18^{* *}$ \\
Grandiose narcissism & $-.32 * *$ & $-.29 * *$ & $.67 * *$ & $.11 *$ & $.26 * *$ & $-.15^{* *}$ & $-.41^{* *}$ & $-.13^{*}$ & $-.41 * *$ & $.30^{* *}$ & $.75^{* *}$ & $.26^{* *}$ & .05 & $.44 * *$ & -.02 \\
\hline
\end{tabular}

AGR Agreeableness, ASR Assertiveness, COM Compassion, CON Conscientiousness, ENT Enthusiasm, EXT Extraversion, IND Industriousness, INT Intellect, $N E U$ Neuroticism, ORD Orderliness, OPE Openness, OPE/INT Openness/Intellect, $P O L$ Politeness, WITH Withdrawal, VOL Volatility

$* p<0.05, * * p<0.01$ 
Table 3 Comparison of the correlation coefficients of grandiose and vulnerable narcissism and the Big Five aspects

\begin{tabular}{|c|c|c|c|c|c|c|c|c|c|c|}
\hline & VOL & WITH & $\mathrm{COM}$ & POL & ENT & ASR & IND & ORD & INT & OPE \\
\hline$z$ & $-8.47 * *$ & $-12.69 * *$ & 0.21 & $-.4 .73 * *$ & $8.36 * *$ & $16.93 * *$ & $5.21 * *$ & -1.91 & $8.58 * *$ & $-2.75 * *$ \\
\hline
\end{tabular}

ASR Assertiveness, COM Compassion, ENT Enthusiasm; IND Industriousness, INT Intellect, ORD Orderliness, OPE Openness, POL Politeness, WITH Withdrawal, VOL Volatility

$* p<0.05, * * p<0.01$

the personality hierarchy. Moreover, in some cases the magnitude of associations with two aspects of a broader domain differed substantially. The differences within personality traits were especially pronounced in grandiose narcissism. First, we observed a high correlation between grandiose narcissism and Assertiveness $(r=0.71)$. Extraversion is believed to reflect sensitivity to reward (Smillie 2013). However, there might be two distinct classes of reward, appetitive rewards, which involve an increase in the probability of achieving a goal, and consummatory, which represent the actual attainment of a goal (DeYoung 2015). The two aspects of Extraversion derive from this distinction: Assertiveness is a reflection of the former class (wanting), whereas Enthusiasm reflects the latter class (liking; DeYoung 2015). The high overlap between grandiose narcissism and Assertiveness would suggest that narcissists are especially sensitive to appetitive rewards, which may underlie their increased agency, and motivation toward social status, dominance, and achievements.

The relationship between grandiose narcissism and Assertiveness may have some consequences for the low Politeness observed among grandiose narcissists. DeYoung (2015) notes that the social behaviors associated with Assertiveness and Enthusiasm are driven by reward processes that are also involved in non-social reward motivation, whereas aspects of Agreeableness are driven by dedicated affiliative bonding and social regulation systems. However, the mechanisms underlying Agreeableness may interact with the goal pursuit process. Interpersonal style and interpretation of the social world have consequences for goal attainment. It is possible that inflated self-views and feelings of superiority to others ("My goals are most important") may predispose grandiose narcissists to more aggressive responses in situations in which someones blocks their goal which in turn leads to a low level of Politeness.

The positive link between grandiose narcissism and Intellect was consistent with our expectations and previous findings showing that grandiose narcissists hold inflated views of their cognitive abilities (Dufner et al. 2012; Gabriel et al. 1994; Zajenkowski and Czarna 2015). The belief that high intelligence is beneficial for individual life success is rooted in both research and social perception of high cognitive ability (Mõttus et al. 2008). Some researchers suggest that positive illusions may serve a self-regulatory function for grandiose narcissists, enabling them to maintain positive feelings (Campbell and Foster 2007).

We also found a moderate positive correlation between Industriousness, an aspect of Conscientiousness, and grandiose narcissism. Industriousness reflects a high need for achievement and ability to pursue non-immediate goals (DeYoung 2015). These characteristics might be valued by narcissists because of their agentic features and the fact that they allow them to reach high social status. Moreover, some data show that grandiose narcissists, indeed, are persistent, but only in some circumstances. For example, individuals scoring high in narcissistic grandiosity put more effort into solving unsolvable tasks but only if that was the only option to amplify positive self-image (Wallace et al. 2009) and they are more likely to invest in achieving a goal that affords personal glory (Woodman et al. 2011).

The current results indicated that vulnerable narcissism, in comparison to grandiose narcissism, showed a more consistent pattern of correlations with personality aspects within Big Five traits. In other words, vulnerable narcissism was associated with less differentiated personality profile. This might be due to the fact that this type of narcissism is more closely linked to psychopathology (e.g. Miller et al. 2011) and, as a consequence, located on relatively extreme personality level.

As expected individuals with high vulnerable narcissism exhibited relatively high levels of both aspects of Neuroticism which is in line with previous investigations (Miller et al. 2018b). Moreover, numerous studies have shown that this type of narcissism is associated with a wide variety of variables encompassing negative emotionality (the core of Neuroticism), including anxiety, depression and vulnerability (Miller et al. 2011), as well as anger and hostility (Krizan and Johar 2015). DeYoung (2015) suggests that Neuroticism primary determines the level at which uncertainty, threat, or punishment triggers a defensive response. Defensive responses might be of two distinct kinds: active defense and passive avoidance. Volatility involves emotional and behavioral responses to immediate threats or punishments, the tendency to be emotionally labile, to get irritated, or angry easily and, thus, reflects the tendency toward active defense. Passive avoidance involves involuntary inhibition of approach toward a goal in response to threat. Our results suggest that individuals scoring high in vulnerable narcissism exhibit both active and passive forms of defensive responses. 
Individuals scoring high on vulnerable narcissism exhibited low levels of both aspects of Extraversion. The negative correlation with Enthusiasm is consistent with previous findings showing that vulnerable narcissists score low on positive affect as well as social avoidance scales (Miller et al. 2011). Additionally, they are rather low on agency and do not express their opinions openly (Lannin et al. 2014), which is congruent with low Assertiveness. In the case of Agreeableness, the (negative) associations with both Compassion and Politeness were rather marginal. Interestingly, in prior work of Miller et al. (2011), vulnerable narcissism correlated mainly (negatively) with the Trust facet of the NEO-PI-R Agreeableness scale. In the Big Five Aspects Scale there are actually no items reflecting trust-related behavior (DeYoung et al. 2007). This might be the reason for the rather small association between vulnerable narcissism and Agreeableness in the present investigation.

Our study revealed a finding which was not previously discussed in the literature. Specifically, we found a positive correlation between vulnerable narcissism and Openness. Although the link between Openness and vulnerable narcissism might seem counterintuitive, the empirical data provide some explanation for this relation. First, vulnerable narcissism has been shown to correlate with a number of psychopathological symptoms including depression, paranoia, psychoticism, and schizotypal personality (Miller et al. 2011). Interestingly, Openness appears to be positively linked to these variables. For instance, Aesthetics and Feelings, the facets of the NEO PI-R that are markers of the Openness aspect, were associated with depression (Wolfenstein and Trull 1997). Moreover, DeYoung (2014) observes that Openness is positively related to Neuroticism. Thus, Openness may contribute to the risk for internalizing disorders by allowing a greater range of stimuli into awareness (DeYoung 2014; DeYoung et al. 2012). Because vulnerable narcissism, Openness and Neuroticism are positively related, one may speculate about whether they share hypervigilance and increased awareness. Furthermore, DeYoung et al. (2012) suggested that Openness might also be close to positive schizotypy, which comprises magical ideation, perceptual aberration, overinclusive thinking. and the erroneous perception of patterns or causal connections. Indeed, positive schizotypy appears to be related to Openness. Apparently, seeing patterns or causal connections where none exist is beneficial for creative behavior, since Openness predicts creativity (DeYoung 2014).

Finally, it is worth mentioning that the two types of narcissism showed quite distinct personality profiles and differed in case of eight Big Five aspects. The biggest differences were associated with Assertiveness and Withdrawal. This corroborates and extends previous findings showing that specific aspects of Extraversion (Assertiveness) and Neuroticism (Withdrawal) differentiate mostly grandiose narcissism and vulnerable narcissism. There were no differences in correlations of the two narcissisms of Orderliness and Compassion. Whereas the first aspect seems to not important for any kind of narcissism, Compassion was negatively associated with both narcissisms. Interestingly, partial correlations showed that the relationships of grandiose and vulnerable narcissism with Compassion remained essentially unchanged when the other narcissism was controlled. This may suggest that there are different mechanisms linking each narcissism with Compassion.

\section{Limitation and Future Research}

The current study explored the relationship between the Big Five aspects and two types of narcissism. The results indicated that the analysis at the aspects level (between narrow facets and broad domains) provides important information about the nature of narcissism, especially in the grandiose version. However, the study has several limitations. First, although the sample size was relatively large (allowing to detect correlations of .14 with power of .85) it consisted of young adults, mostly undergraduated students. Thus it remains an open question to what extent the findings generalize to other populations (e.g. older people). Second, we used only measures of narcissism and personality which allowed us to explore simple associations. However, it would be valuable to see how personality aspects account for narcissism's relations with external variables. Another problem is associated with the instrument measuring Big Five aspects. Previous research indicated that some of the aspects are highly correlated across domains. For instance, in the original study, it was found that Enthusiasm and Compassion, as well as Assertiveness and Intellect show correlation coefficients almost .60. Likewise, in the current investigation we obtained high associations (exceeding .50) of aspects from different domains. These findings suggest that perhaps further research and analyses are required to establish final lower-order factors of Big Five.

Although some robust associations have been found, more studies are necessary to examine to what extent some aspects of personality explain the associations of narcissism with external variables. For instance, we found a high correlation between grandiose narcissism and Assertiveness. It is suggested that Extraversion is related to dopamine (Smillie 2013), however, Assertiveness is more closely linked to dopamine than Enthusiasm and dopamine is regarded as the major neurotransmitter for incentive reward and a driver of exploration (DeYoung 2015). Given the high association between Assertiveness and grandiose narcissism, one may wonder to what extent these constructs share a biological mechanism and both correlate with dopamine. Additionally, grandiose narcissism was relatively highly associated with Intellect, which reflects perceived intelligence, but also intellectual curiosity (DeYoung 2014). It would be interesting to see whether narcissists manifest increased engagement in cognitive activities similar to individuals scoring high on Intellect. In a 
similar vein, one may consider potential consequences (e.g. for creativity) of the fact that Openness and vulnerable narcissism share some common features.

Furthermore, the existing research literature focused on the differential associations of the two narcissisms with the Big Five subdimensions. However, recent findings suggest that other personality conceptualizations, such as the HEXACO model, might be relevant for antisocial traits. Specifically, it has been shown that the Dark Triad (narcissism, Machiavellianism, psychopathy) latent factor overlaps essentially with the Honesty-Humility factor from the HEXACO scale (Hodson et al. 2018). It needs to be acknowledged, however, that in the Dark Triad concept, narcissism is mainly understood in the grandiose version and in the study by Hodson et al. (2018) its highest correlation was with the Modesty subscale of Honesty-Humility (see also Visser 2018). It remains an open question how vulnerable narcissism would be associated with Honesty-Humility and its subscales. One would expect, for instance, relatively high negative correlation with the Sincerity subscale, since there is evidence that vulnerable narcissists are less genuine in interpersonal relations (e.g. Lannin et al. 2014).

\section{Conclusion}

In the current study we found large differences in personality profiles of grandiose and vulnerable narcissism. Specifically, grandiose narcissism correlated mainly with aspects related to agency, both social (high Assertiveness), and personal (high Intellect), as well as rivarly (low Politeness) and low anxiety (low Withdrawal), By contrast, vulnerable narcissism showed less differentiated correlations within broad domains and was primary associated with a wide range of negative emotionality including tendency toward anxiety (high Withdrawal) and anger (high Volatility).

Acknowledgments This work was supported from a grant no 2016/23/B/ HS6/00312 funded by National Science Centre in Poland.

Funding This work was funded by National Science Centre in Poland (grant number 2016/23/B/HS6/00312).

Compliance with Ethical Standards All procedures performed in studies involving human participants were in accordance with the ethical standards of the institutional research committee and with the 1964 Helsinki declaration and its later amendments or comparable ethical standards. Informed consent was obtained from all individual participants included in the study. This article does not contain any studies with animals performed by any of the authors.

Conflict of Interest The authors declare that there are no potential conflicts of interest with respect to the research, authorship, and/or publication of this article.
Open Access This article is distributed under the terms of the Creative Commons Attribution 4.0 International License (http:// creativecommons.org/licenses/by/4.0/), which permits unrestricted use, distribution, and reproduction in any medium, provided you give appropriate credit to the original author(s) and the source, provide a link to the Creative Commons license, and indicate if changes were made.

Publisher's Note Springer Nature remains neutral with regard to jurisdictional claims in published maps and institutional affiliations.

\section{References}

Bazińska, R., \& Drat-Ruszczak, K. (2000). The structure of narcissism measured with polish adaptation of NPI. Czasopismo Psychologiczne, 6, 171-187.

Campbell, W., \& Foster, J. (2007). The narcissistic self: Background, an extender agency model, and ongoing controversies. In C. Sedikides \& S. Spencer (Eds.), Frontiers in social psychology: The self (pp. 115-138). Philadelphia: Psychology Press.

Chamorro-Premuzic, T., \& Furnham, A. (2004). A possible model to understand the personality-intelligence interface. British Journal of Psychology 95, 249-264.

Costa, P., \& McCrae, R. (1992a). Revised NEO personality inventory manual. Odessa: PAR.

Costa, P., \& McCrae, R. (1992b). Trait psychology comes of age. In T. Sonderegger (Ed.), Nebraska symposium on motivation: Psychology and aging (pp. 169-204). Lincoln: University of Nebraska Press.

Czarna, A., Dufner, M., \& Clifton, A. (2014). The effects of vulnerable and grandiose narcissism on liking-based and disliking-based centrality in social networks. Journal of Research in Personality, 50, $42-45$.

Czarna, A., Leifeld, P., Śmieja, M., Dufner, M., \& Salovey, P. (2016). Do narcissism and emotional intelligence win us friends? Modeling dynamics of peer popularity using inferential network analysis. Personality and Social Psychology Bulletin, 42, 1588-1599.

Czarna, A., Zajenkowski, M., \& Dufner, M. (2018). How does it feel to be a narcissist? Narcissism and emotions. In T. Hermann, A. Brunell, \& J. Foster (Eds.), Handbook of trait narcissism: Key advances, research methods, and controversies. New York: Springer.

DeYoung, C. (2014). Openness/intellect: A dimension of personality reflecting cognitive exploration. In M. Cooper \& R. Larsen (Eds.), APA handbook of personality and social psychology (pp. 369-399). Washington: APA.

DeYoung, C. (2015). Cybernetic Big Five theory. Journal of Research in Personality, 56, 33-58.

DeYoung, C., Quilty, L., \& Peterson, J. (2007). Between facets and domains: 10 aspects of the Big Five. Journal of Personality and Social Psychology, 93, 880-896.

DeYoung, C. G., Shamosh, N. A., Green, A. E., Braver, T. S., \& Gray, J. R. (2009). Intellect as distinct from openness: Differences revealed by fMRI of working memory. Journal of Personality and Social Psychology, 97, 883-892.

DeYoung, C., Grazioplene, R., \& Peterson, J. (2012). From madness to genius: The openness/intellect trait domain as a paradoxical simplex. Journal of Research in Personality, 46, 63-78.

Digman, J. (1997). Higher-order factors of the Big Five. Journal of Personality and Social Psychology, 73, 1246-1256.

Dufner, M., Denissen, J., van Zalk, M., Matthes, B., et al. (2012). Positive intelligence illusions: On the relation between intellectual selfenhancement and psychological adjustment. Journal of Personality, 80, 537-571. 
Gabriel, M., Critelli, J., \& Ee, J. (1994). Narcissistic illusions in selfevaluations of intelligence and attractiveness. Journal of Personality, 62, 143-155.

Goldberg, L. (1992). The development of markers for the Big-Five factor structure. Psychological Assesment, 4(1), 26-42.

Hart, W., Adams, J., Burton, K., \& Tortoriello, G. (2017). Narcissism and self-presentation: Profiling grandiose and vulnerable Narcissists' self-presentation tactic use. Personality and Individual Differences, $104,48-57$.

Hendin, H. M., \& Cheek, J. M. (1997). Assessing hypersensitive narcissism:a reexamination of Murray's narcissism scale. Jourlan of Research in Personality, 31, 588-599.

Hodson, G., Book, A., Visser, B. A., Volk, A. A., Ashton, M. C., \& Lee, K. (2018). Is the dark triad common factor distinct from low honesty-humility? Journal of Research in Personality, 73, 123-129.

Jang, K. L., Hu, S., Livesley, W. J., Angleitner, A., Riemann, R., \& Vernon, P. A. (2002). Genetic and environmental influences on the covariance of facets defining the domains of the five-factor model of personality. Personality and Individual Differences, 33, 83-101.

Jauk, E., Weigle, E., Lehmann, K., Benedek, M., \& Neubauer, A. C. (2017). The relationship between grandiose and vulnerable (hypersensitive) narcissism. Frontiers in Psychology, 8, 1600.

Krizan, Z., \& Johar, O. (2015). Narcissistic rage revisited. Journal of Personality and Social Psychology, 108, 784-801.

Lannin, D., Guyll, M., Krizan, Z., \& Madon, S. (2014). When are grandiose and vulnerable narcissists least helpful? Personality and Individual Differences, 56, 127-132.

McCrae, R. R. (2008). A note on some measures of profile agreement. Journal of Personality Assessment, 90, 105-109.

Miller, J., Hoffman, B., Gaughan, E., Gentile, B., Maples, J., \& Campbell, W. (2011). Grandiose and vulnerable narcissism: A nomological network analysis. Journal of Personality, 79, 1013-1042.

Miller, J. D., Gentile, B., Carter, N. T., Crowe, M., Hoffman, B. J., \& Campbell, W. K. (2018a). A comparison of the nomological networks associated with forced-choice and Likert formats of the narcissistic personality inventory. Journal of Personality Assessment, 100, 259-267.

Miller, J. D., Lynam, D. R., Vize, C., Crowe, M., Sleep, C., MaplesKeller, J. L., et al. (2018b). Vulnerable narcissism is (mostly) a disorder of neuroticism. Journal of Personality, 86, 1467-1494.
Mõttus, R., Allik, J., Konstabel, K., Kangro, E., \& Pullmann, H. (2008). Beliefs about the relationships between personality and intelligence. Personality and Individual Differences, 45(6), 457-462.

Pincus, A., Ansell, E., Pimentel, C., Cain, N., Wright, A., \& Levy, K. (2009). Initial construction and validation of the pathological narcissism inventory. Psychological Assessment, 21, 365-379.

Raskin, R., \& Hall, C. (1979). A narcissistic personality inventory. Psychological Reports, 45, 590.

Sedikides, C., Rudich, E., Gregg, A., Kumashiro, M., \& Rusbult, C. (2004). Are normal narcissists psychologically healthy? Selfesteem matters. Journal of Personality and Social Psychology, 87, 400- 416.

Smillie, L. (2013). Extraversion and reward processing. Current Directions in Psychology Science, 22, 167-172.

Steiger, J. (1980). Tests for comparing elements of a correlation matrix. Psychological Bulletin, 87(2), 245-251.

Strus, W., Cieciuch, J., \& Rowiński, T. (2014). Polish version of the Big Five aspects scales. Bydgoszcz: Polish Psychological Association meeting.

Visser, B. A. (2018). Narcissism and the Big Five/HEXACO models of personality. In A. Hermann, A. Brunell, \& J. Foster (Eds.), The handbook of trait narcissism: Key advances, research methods, and controversies. Berlin: Springer.

Wallace, H., Ready, C., \& Weitenhagen, E. (2009). Narcissism and task persistence. Self and Identity, 8, 78-93.

Wolfenstein, M., \& Trull, T. (1997). Depression and openness to experience. Journal of Personality Assessment, 69, 614-632.

Woodman, T., Roberts, R., Hardy, L., Callow, N., \& Rogers, C. H. (2011). There is an "I" in TEAM. Research Quarterly for Exercise and Sport, 82(2), 285-290.

Zajenkowski, M., \& Czarna, A. (2015). What makes narcissists unhappy? Subjectively assessed intelligence moderates the relationship between narcissism and psychological well-being. Personality and Individual Differences, 77, 50-54.

Zajenkowski, M., \& Matthews, G. (2019). Intellect and openness differentially predict affect: Perceived and objective cognitive ability contexts. Personality and Individual Differences, 137, 1-8.

Zajenkowski, M., Witowska, J., Maciantowicz, O., \& Malesza, M. (2016). Vulnerable past, grandiose present: The relationship between vulnerable and grandiose narcissism, time perspective and personality. Personality and Individual Differences, 98, 102-106. 